\title{
Removing Imaging Distortions Through Automatic Stitching of EBSD Mosaics
}

\section{David J. Rowenhorst}

\section{The US Naval Research Laboratory, Materials Science Division, Washington, DC}

Electron Backscatter Diffraction (EBSD) has become an extremely popular electron microscopy, allowing for the direct measurement the crystal orientation of metallic and ceramic systems. Recent advances in the acquisition hardware and software have allowed for much larger datasets to be collected in reasonable timeframes, allowing for the collection crystallographic maps that create an image of the underlying microstructure based on the crystallographic orientation at each point. For many analyses it is highly advantageous to collect a larger region of interest than would fit within one field of view in the Scanning Electron Microscope (SEM).

Through the use of stage translations within the SEM, a mosaic image can be built up to cover a much larger area than the field of view. Under ideal conditions, the stage movements would have enough accuracy to make perfectly aligned images at the tile edges, and for relatively low spatial resolution scans with minimal image distortions it has already been demonstrated how very large mosaic scans can be collected when using custom SEM control software and using overlapping image tiles[1, 2]. EBSD data collection must be collected with the sample at a high angle of tilt (typically $70^{\circ}$ ), which is far from the ideal imaging conditions of most SEMs, and consequently there are often significant imaging distortions within each field of view, thus the image tiles of a mosaic cannot overlap. Even if the electron-optics of the SEM are well calibrated with minimal distortions, even small sample alignment errors can introduce significant imaging artifacts that can be difficult to detect during data collection[3].

The technique presented here provides an algorithm that removes these image distortions while simultaneously producing a unified EBSD dataset by stitching overlapping mosaic tiles. Additionally, the algorithm requires minimal additional knowledge or effort from the EBSD user during collection or post processing and allows for the use of standard instrumentation and data collection methods. Data collection involves collecting a set of overlapping EBSD data scans that are related by stage translations. Using EDAX/TSL EBSD 6.x software, this is referred to as a "Combo scan", where the user sets a fractional overlap of each tile, typically between $10-15 \%$, and the data collection software will collect an EBSD scan at each programmed stage location.

The distortion removal program, (written in IDL, from Exelis Visual Information Systems Inc) requires the user to provide the approximate overlap fraction for the tiles, and the number of rows and columns for the mosaic image. The algorithms then reads in each tile and creates an Inverse Pole Figure (IPF) map for each tile (See Figure 1). The algorithm, by use of a Nelder-Mead optimatization method, attempts to maximize the degree of overlap between the IPF images by applying a polynomial warping function to the image tiles. The assumption is that the image distortions are invariant with stage translations; therefore each image contains the same exact image distortion, and can be corrected by the same warping function applied to each tile. The degree of overlap for each set of tiles is determined by the summing maximum value of the cross-correlation between the two overlapping. The optimization routine adjusts the coefficients of the polynomial function until a local maximum in the sum of the maximum value of the cross-correlations is found. Once this local maximum is determined, the warping polynomial is applied to each EBSD with nearest neighbor interpolation used to fill in the data points. 
The undistorted data is then stitched into a unified dataset using the cross-correlations of the overlapping regions to determine the exact translations between each tile, similar to the method described in [2].

Figure 1 shows the IPF maps of a duplex stainless steel. Before scanning, the sample was laser etched to mark out the region of interest, creating a $1 \mathrm{~mm} \times 1 \mathrm{~mm}$ frame around the area. The mosaic is made up of a 2 row x 2 column mosaic with approximately 10\% overlap between the images. The step size of each scan was $1 \mu \mathrm{m}$, with a nominal tile size of $500 \times 500 \mu \mathrm{m}$. Figure 1a shows the original images. The tiles are laid out as to maximize the amount of overlap at the center of the image. It is apparent along the seams of the tiles that at the outer edges the tiles do not match. Figure $1 \mathrm{~b}$ shows the IPF combined with the "Image Quality" parameter to highlight the seam locations. Here we show that the microstructure has near perfect matching across the seams.

We believe that this algorithm has the potential to allow for much larger areas to be analyzed, leading to better statistical evaluations of microstructure as while maintaining a high-resolution image. Also this technique can be easily combined with the mosaic techniques that implement advanced instrumentation to further improve this data collection method [4].

[1] AR Shiveley, et al, Journal of Microscopy 244 (2011) p. 181

[2] AL Pilchak, et al, Journal of Microscopy 248 (2012), p. 172

[3] G. Nolze, Ultramicroscopy 107 (2007), p. 172

[4] The authors gratefully acknowledge the support of ONR Alloy and Joining Program under W. Mullins and the support of the US Naval Research Lab.
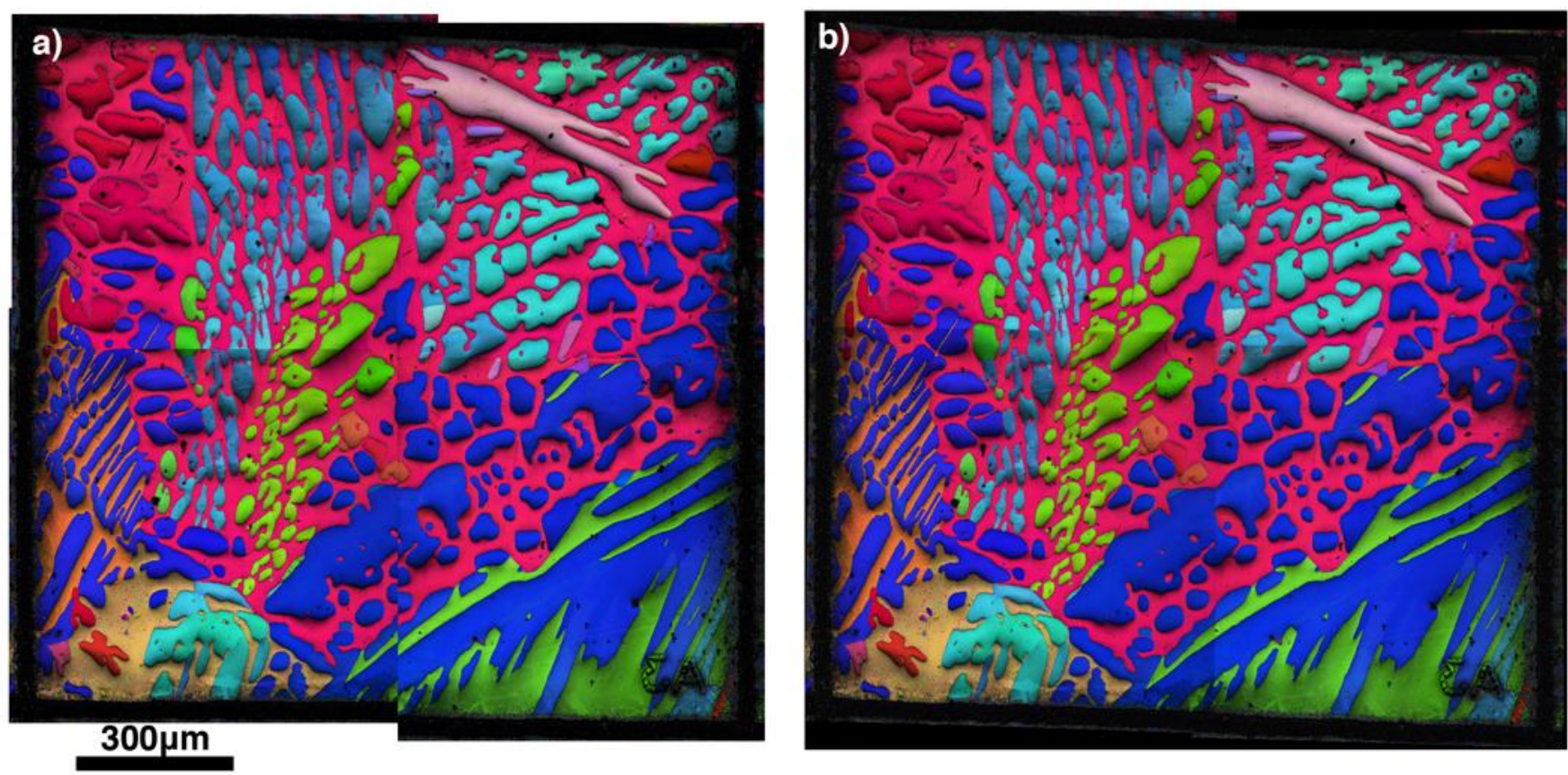

Figure 1. Mosaic ( 2 columns by 2 rows) EBSD IPF image of a duplex stainless steel, (a) shows the original tiles, showing poor overlap at the seams of the images. (b) Is the same scan after the image distortions have been removed by maximizing the cross-correlation of the overlapping areas. 